\title{
Time-course effects of functional fitness sessions performed at different intensities on the metabolic, hormonal, and BDNF responses in trained men
}

\author{
Ramires Alsamir Tibana ${ }^{1 *}$, Ivo Vieira de Sousa Neto², Nuno Manuel Frade de Sousa ${ }^{3}$, \\ Wellington Martins dos Santos ${ }^{4}$, Jonato Prestes ${ }^{4}$, João Henrique Falk Neto ${ }^{5}$, Fábio H. Dominski ${ }^{6}$, \\ Michael D. Kennedy ${ }^{5}$ and Fabricio Azevedo Voltarelli ${ }^{1}$
}

\begin{abstract}
Background: To investigate the time-course effects of a self-regulated training session (performed at an rating perceived exertion of 6/10), all-out session, and a control session on the metabolic, hormonal, and brain derived neurotrophic factor (BDNF) responses in Functional-Fitness (FFT) participants.

Methods: In a randomized, crossover fashion, eight healthy males (age $28.1 \pm 5.4$ years old; body mass $77.2 \pm 4.4 \mathrm{~kg}$; $\mathrm{VO}_{2 \max }: 52.6 \pm 4.6 \mathrm{~mL} .(\mathrm{kg} \cdot \mathrm{min})^{-1} ; 2000 \mathrm{~m}$ rowing test $7.35 \pm 0.18 \mathrm{~min} ; 1 \mathrm{RM}$ back squat $135.6 \pm 21.9 \mathrm{~kg}$ ) performed a FFT session under two different conditions: all-out, or with the intensity controlled to elicit an rating perceived exertion (RPE) of 6 in the Borg 10-point scale (RPE6). A control session (no exercise) was also completed. Metabolic (lactate and creatine kinase), hormonal (testosterone and cortisol), and BDNF responses were assessed pre, post- $0 \mathrm{~h}, 1 \mathrm{~h}, 2 \mathrm{~h}$ and $24 \mathrm{~h}$ after the sessions.

Results: Creatine kinase concentrations were significantly higher $(p \leq 0.05)$ after $24 \mathrm{~h}$ for both training sessions. Total and free testosterone concentrations were lower post- $2 \mathrm{~h}$ for all-out when compared to the RPE6 session $(p \leq 0.05)$. Serum cortisol concentration increased post-0 h $(p=0.011)$ for RPE6 and post-0 h $(p=0.003)$ and post- $1 \mathrm{~h}(p=0.030)$ for all-out session when comparing to baseline concentrations. BDNF was significantly higher $(p=0.002)$ post- $0 \mathrm{~h}$ only for the all-out session when compared to baseline. A positive correlation between blood lactate concentrations and BDNF ( $r=0.51 ; p=0.01)$ was found for both effort interventions.
\end{abstract}

Conclusions: A single FFT session when performed in all-out format acutely increases the concentrations of serum BDNF. However, physiological stress markers show that the all-out session requires a longer recovery period when compared to the RPE6 protocol. These findings can be helpful to coaches and practitioners design FFT session.

Keywords: CrossFit, High-intensity functional training, Rating perceived exertion, Endocrine system, Metabolism, Neurotrophin

*Correspondence: ramirestibana@gmail.com

${ }^{1}$ Graduate Program in Health Sciences, Faculty of Medicine, Federal University of Mato Grosso (UFMT), Cuiabá, Brazil

Full list of author information is available at the end of the article

\section{Introduction}

Functional fitness training (FFT) (a.k.a. CrossFit) involves the performance of exercises that comprise whole body, and that are executed in multiple planes of motion [1]. The FFT sessions can be designed to original author(s) and the source, provide a link to the Creative Commons licence, and indicate if changes were made. The images or other third party material in this article are included in the article's Creative Commons licence, unless indicated otherwise in a credit line to the material. If material is not included in the article's Creative Commons licence and your intended use is not permitted by statutory regulation or exceeds the permitted use, you will need to obtain permission directly from the copyright holder. To view a copy of this licence, visit http://creativecommons.org/licenses/by/4.0/. The Creative Commons Public Domain Dedication waiver (http://creativeco mmons.org/publicdomain/zero/1.0/) applies to the data made available in this article, unless otherwise stated in a credit line to the data. 
challenge various physiological systems at the same time [2], through the use of gymnastics, weightlifting, and cardiovascular exercises [3], and thus, have been shown to improve multiple fitness components concomitantly. Practitioners commonly perform 3-5 whole body training sessions per week, and while the selection of exercises depends on which fitness components are being targeted [1], most sessions dedicate a period of time to metabolic conditioning [4].

One of the potential reasons why the physiological responses to metabolic conditioning of FFT are not yet well understood lies in the fact that the sessions can vary significantly. The protocols might differ in their duration (2-30 $\mathrm{min}$ ), exercise modality and selection (cardiovascular, gymnastic, and/or weightlifting exercises), method [for time or as many rounds as possible (AMRAP)], and intensity (absolute or relative load) [5]. Many of these sessions are performed as all-out efforts, where the goal is to complete the task in the shortest amount of time possible or to complete the highest amount of work in a set period of time $[2,6,7]$. As many of these sessions are performed at a high intensity, previous research has shown that the metabolic conditioning sessions of FFT resulted in increased acute oxidative stress [8]; high metabolic, inflammatory [3], and cardiovascular responses; elevated perceived exertion [9]; and increased sympathetic nervous system markers (i.e., epinephrine and norepinephrine) [10]. As a result of the increases in oxidative and inflammatory markers, and the extreme effort associated with FFT, some studies have raised concerns about a tendency for the development of symptoms of overtraining in functional fitness practitioners [8]. To address this issue, previous studies have demonstrated the effectiveness of utilizing the rating of perceived exertion (RPE) to control the intensity of these sessions, and consequently, the physiological responses to exercise during [4] and after [2] a FFT session.

The distinct modes, intensity, and duration of exercises that can be manipulated in FFT sessions, along with differences in individual responsiveness to the sessions, may lead to different hormonal responses post-FFT sessions [11]. Such changes in hormonal profiles following a session might have important implications. Previous studies have revealed that long periods of hormonal disturbances are likely to lead to impairments in performance, inflammatory conditions, and increased muscle fatigability [1214]. Similarly, while transient increases in creatine kinase, and cortisol immediately following a training session are expected, chronically high levels are not desirable [4]. In this context, changes in hormonal responses can provide important information in detecting early signs of non-functional overreaching. Understanding the timecourse endocrine response to FFT sessions performed at different intensities, therefore, might assist in ensuring optimal training prescription.

In addition to its effects on hormonal concentrations, FFT has been demonstrated to elicit important alterations in other biomarkers. The brain derived neurotrophic factor (BDNF) is a key biomarker that stimulates neurogenesis, neuron survival, and modulates the differentiation of cells developed in the hippocampus, which can be essential for cognition, memory [15] and consequently neuroplasticity improvement [16]. Murawska-Cialowicz et al. [17] revealed that 3 months of FFT training resulted in a significant increase in resting BDNF levels in male and female participants. Indeed, a single FFT session is a potent stimulus that leads to an acute increase in serum BDNF concentrations [18]. Of interest, prior study has demonstrated that blood lactate produced during exercise is correlated with BDNF production [19] and recently a systematic review with metaanalysis considered that FFT session normally causes a substantial metabolic stress, leading to metabolite accumulation (e.g., lactate up to $18 \mathrm{mmol} / \mathrm{L}$ ) [20]. Therefore, understanding the time-course of changes in peripheral BDNF levels and the possible relationship with lactate following FFT sessions performed at different intensities is an important pursuit.

Considering that the adaptations elicited during a training program result from the summation of training bouts, understanding the role that the intensity of FFT protocols have on neurotrophin, metabolic and hormonal biomarkers in a time-dependent manner is an important step to understand these adaptive mechanisms. Specifically, this might assist in clarifying the key mediators by which FFT exerts beneficial or maladaptive effects. Assessing the response of different biomarkers to the training stimulus might help in understanding the overall effectiveness of FFT sessions performed at distinct intensities, and therefore, assist in optimizing training programs. Thus, the purpose of the present study was to investigate the acute time-course of metabolic, hormonal and BDNF responses following two FFT sessions performed at different intensities (RPE6 and all-out). It is hypothesized that a higher intensity will lead to a more pronounced hormonal and metabolic response, with the lower intensity session (RPE6) showing a reduced magnitude of endocrine response to an FFT session.

\section{Methods \\ Subjects}

Eight male subjects (age 28.1 \pm 5.4 years old; body mass $77.2 \pm 4.4 \mathrm{~kg} ; \mathrm{VO}_{2 \max }: 52.6 \pm 4.6 \mathrm{~mL}$.(kg.min) ${ }^{-1}$; $2000 \mathrm{~m}$ rowing test: $7.35 \pm 0.18 \mathrm{~min} ; 1 \mathrm{RM}$ back squat: $135.6 \pm 21.9 \mathrm{~kg}$ ) were recruited. All subjects were free of injury or known illnesses, were not using performance 
enhancing drugs, and had more than 12 months of FFT experience (3.8 \pm 1.4 years, $1.5-6$ years of experience). Participants were advised to sleep six to eight hours the night before the tests, maintain regular nutritional and hydration habits, avoid intense exercise $48 \mathrm{~h}$ prior to the sessions, as well as avoid smoking, alcohol, and caffeine consumption $24 \mathrm{~h}$ before a session. All subjects provided informed consent, and the study was approved by the University Research Ethics Committee for Human Use (2.698.225/Universidade Estácio de Sá/UNESA/RJ and ethics ID Pro00110581) and conformed to the Helsinki Declaration on the use of human participants for research.

\section{Experimental design}

In this study, the participants performed a FFT session under two different conditions, either with the intensity controlled based on an RPE of 6 (RPE6) or as an all-out effort (all-out), in addition to a control session $(\mathrm{CON})$. The metabolic and hormonal responses to the different conditions were the dependent variables. These responses were assessed prior to the start of the session, immediately post, and $1 \mathrm{~h}, 2 \mathrm{~h}$ and $24 \mathrm{~h}$ after the sessions, to compare the acute effects of a metabolic conditioning FFT session performed with different intensities (Fig. 1).

The subjects completed a metabolic conditioning training session (five to seven days apart) in a randomized fashion under two different conditions: (a) all-out or (b) intensity-controlled, based on an RPE6 (hard) on a modified version of the Borg CR-10 scale (RPE6) [2]. A control session, where the participants were instructed to spend $22 \mathrm{~min}$ in a sitting position, without any type of exercise, was also performed. The metabolic conditioning training session was the Tibana Test [2], which involved the completion of four different bouts of work, each separated by 2 min of rest (Fig. 2). The rounds consisted of 4 min of as many rounds as possible (AMRAP) of five thrusters $(60 \mathrm{~kg})$ and 10 box jumps over (round 1); 4 min of AMRAP of 10 power clean $(60 \mathrm{~kg})$ and 20 pullups (round 2); 4 min of AMRAP of 15 shoulder to overhead $(60 \mathrm{~kg})$ and 30 toes to bar (round 3); and $4 \mathrm{~min}$ of AMRAP of 20 calories of rowing and 40 wall ball $(9 \mathrm{~kg}$; round 4).

During the all-out condition, the subjects were instructed to complete the maximum number of repetitions possible for each round. In the RPE6 condition, they performed the same conditioning session, but were

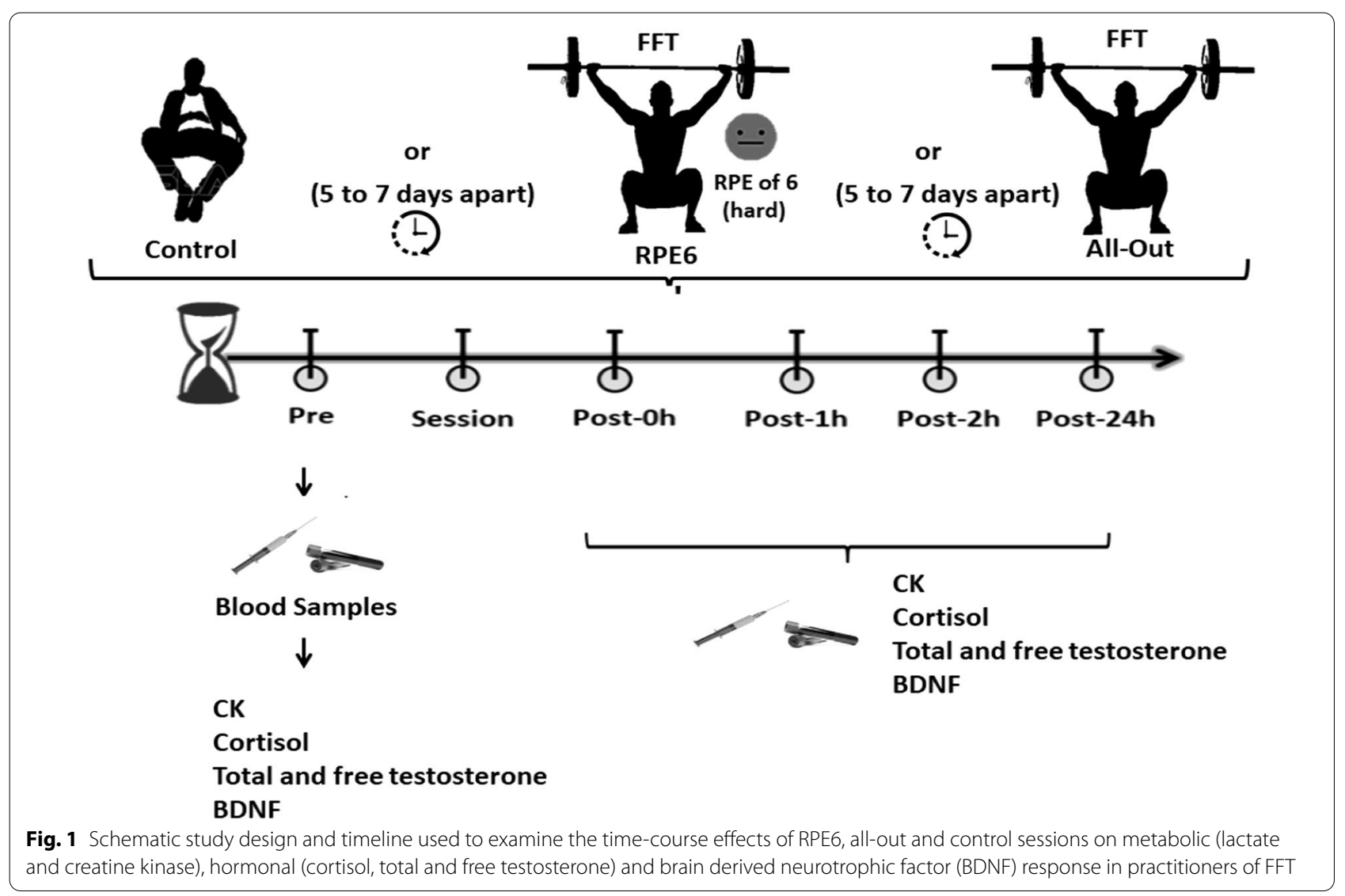




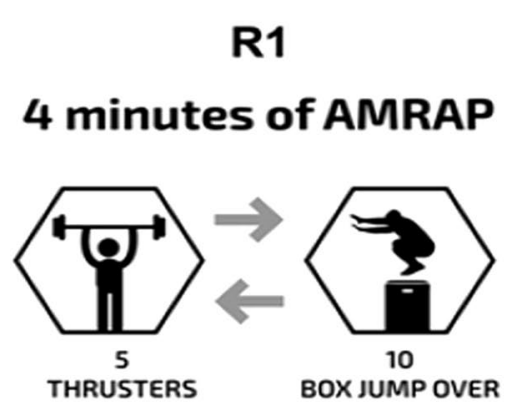

4 minutes of AMRAP

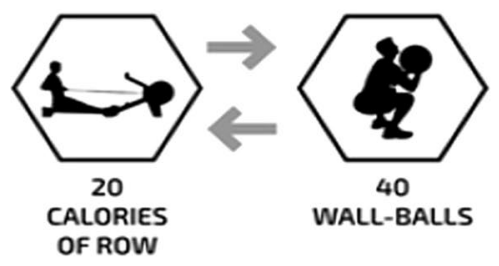

R4
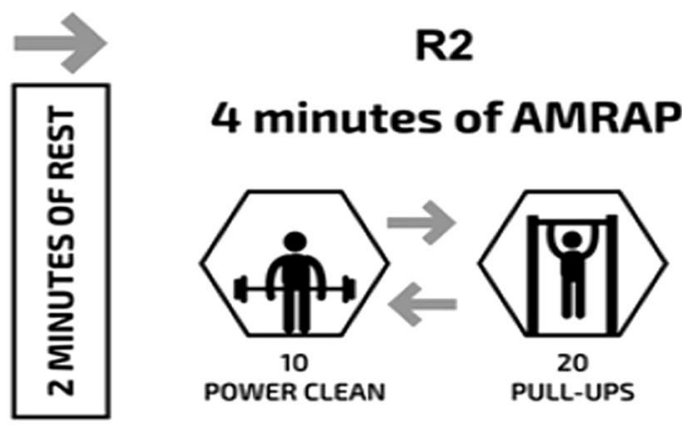

2 MINUTES OF REST

\section{4 minutes of AMRAP}

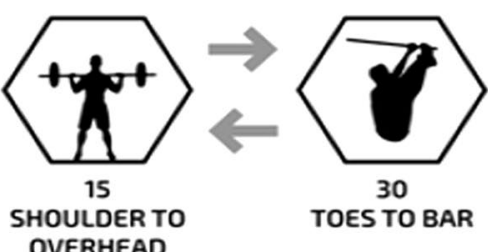

R3

Fig. 2 Description of the metabolic conditioning sessions (Tibana Test). AMRAP, as many rounds as possible

told to self-regulate the intensity of their effort based on a perception of effort of 6 out of 10, on an adapted version of the Borg CR-10 scale [21, 22]. To achieve this, subjects were instructed to take more breaks if needed, or to pace themselves in the execution of their exercises to keep the perception of effort at the desired level. No changes to the weights were performed during the sessions. The adapted Borg CR-10 scale was printed and available to the participants as a visual reminder of the prescribed target intensity. This strategy has been shown to be successful in ensuring the athletes perform their FFT sessions within the desired intensity, based on previously published work $[2,7]$. During each metabolic condition training session and the control session, blood samples were collected from the antecubital vein to analyze changes in the concentrations of creatine kinase, cortisol, total testosterone, free testosterone and BDNF.

\section{Blood samples collected, hormonal and BDNF analysis}

Blood samples were collected immediately before (pre) and immediately (post- 0 h), $1 \mathrm{~h}$ (post- $1 \mathrm{~h}$ ), $2 \mathrm{~h}$ (post- $2 \mathrm{~h}$ ) and $24 \mathrm{~h}$ (post-24 h) after the FFT and control sessions by venipuncture from the antecubital vein. The samples were collected in 5-mL evacuated tubes (Vacutainer; Becton, Dickinson and Company, Franklin Lakes, NJ, USA). The tubes were refrigerated for $1 \mathrm{~h}$ and samples were centrifuged at $2.900 \mathrm{RPM}$ for $15 \mathrm{~min}$ at $4{ }^{\circ} \mathrm{C}$, and the resultant serum divided into several aliquots, and frozen at $-80{ }^{\circ} \mathrm{C}$ until analysis. Hormonal analyses were performed using a commercial PCR kit lot 167404 (Roche), specific for humans in an automated device (Cobas E601-Roche) for PCR by the electrochemiluminescence method. Serum was analyzed for BDNF using a commercially available enzyme-linked immunosorbent assay (ELISA) kit according to manufacturer's instructions (MyBioSource Inc., San Diego, CA, USA). All samples were determined in duplicate to guarantee the precision of the results. Detection range of this method was $31.25-2000 \mathrm{pg} / \mathrm{mL}$, sensitivity of this methods was $18.75 \mathrm{pg} / \mathrm{mL}$, with an intraassay coefficient of $4 \%$ and an inter-assay coefficient of $8 \%$. All analyses were performed in the Immune Gerontology/ Molecular Biology Laboratory of Applied Exercise at the University.

\section{Creatine kinase analysis}

Whole-blood creatine kinase activity was assessed from a single fingertip capillary sample with the subject in a seated position. After pre-warming the hand, a sample of blood $(30 \mu \mathrm{L})$ was obtained and analyzed using a colorimetric assay procedure (Reflotron, Boehringer Mannheim, Germany). Before each testing session, quality control (calibration) measurements were undertaken 
according to the manufacturer's recommendations. The "normal" reference range for creatine kinase activity, as provided by the manufacturer, is $24-195 \mathrm{U} / \mathrm{L}$.

\section{Blood lactate}

Standard procedures were followed for blood lactate collection, management, and analysis according to Falk Neto et al. [2]. Capillary blood samples were collected through a transcutaneous puncture on the medial side of the tip of the middle finger using a disposable hypodermic lancet. Blood lactate concentration was determined by photometric reflectance on a validated Portable Accutrend Plus system (Roche, Sao Paulo, Brazil).

\section{Statistical analysis}

The data are presented as means and 95\% confidence intervals (CI). Shapiro-Wilk tests were applied to assess the normal distribution of the variables assessed. In case of non-normal distribution, the variables were log transformed before analysis. Repeated measures ANOVA was used to compare creatine kinase, and hormonal status between the FFT sessions and the control session. Tukey's post-hoc test was applied in the event of a significant main effect. Repeated measures ANOVA was also used to compare creatine kinase and hormonal concentrations between pre- values and post-FFT sessions in different time points. Lastly, percentage of change from baseline of creatine kinase, and hormonal concentrations was calculated for the different time points and repeated measures ANOVA was also used to compare creatine kinase, and hormonal changes between functional fitness sessions and control session. Cohen's d effect sizes (ES) were calculated using the Cohen's convention [23] to evaluate the magnitude of the change of creatine kinase and hormonal concentrations during the functional fitness and control sessions $(\mathrm{ES} \leq 0.20$ represents a small ES; 0.50 a moderate ES; 0.80 a large effect size). Simple Pearson's $\mathrm{r}$ correlations were used to determine the associations between the hormonal responses and blood lactate concentration and RPE after the FFT sessions and the control condition. The magnitude of the correlations was classified as: $\mathrm{r} \leq 0.1$ trivial; $0.1<\mathrm{r} \leq 0.3$ small; $0.3<\mathrm{r} \leq 0.5$ moderate; $0.5<\mathrm{r} \leq 0.7$ large; $0.7<\mathrm{r} \leq 0.9$ very large; $\mathrm{r}>0.9$ almost perfect [24]. The level of significance was $p \leq 0.05$ and SPSS version 20.0 (Somers, NY, USA) software was used.

\section{Results}

Participants completed a greater number of repetitions (214.4 \pm 18.6 repetitions) during the all-out session when compared to the RPE6 session (190.5 \pm 12.5 repetitions). Blood lactate concentration and RPE were also higher after the all-out session $(18.9 \pm 3.9 \mathrm{mmol} / \mathrm{L}$; RPE: $9.6 \pm 0.7)$ than the RPE6 session $(12.8 \pm 3.2 \mathrm{mmol} / \mathrm{L}$; RPE: $6.2 \pm 0.8)$. An in-depth discussion of these results and its implications has already been published [4].

Table 1 presents the hormonal responses pre- and post-functional fitness sessions. Considering the baseline values, only the cortisol concentration pre- all-out session was greater $(p=0.048)$ than the control session. Creatine kinase concentration was greater $0 \mathrm{~h}(p=0.038)$, $1 \mathrm{~h}(p=0.010), 2 \mathrm{~h}(p=0.011)$ and $24 \mathrm{~h}(p=0.041)$ after the RPE6 and $0 \mathrm{~h}(p=0.001), 1 \mathrm{~h}(p=0.002), 2 \mathrm{~h}$ $(p=0.015)$ and $24 \mathrm{~h}(p=0.058)$ after the all-out sessions. When compared to baseline, serum cortisol concentration was greater post- $0 \mathrm{~h}(p=0.011)$ for RPE6 and post- $0 \mathrm{~h}(p=0.003)$ and post- $1 \mathrm{~h}(p=0.030)$ for all-out session. For the all-out session, cortisol concentration post-24 h was significantly less $(p=0.010)$ than pre- values. Total testosterone and free testosterone were significantly greater $(p \leq 0.05)$ post- $0 \mathrm{~h}$ for RPE6 $(\mathrm{p}=0.007$ for total testosterone and $p=0.010$ for free testosterone) and all-out ( $p=0.005$ for total testosterone and $p=0.003$ for free testosterone) sessions when comparing to baseline concentrations. However, total testosterone and free testosterone were significantly lower $(p=0.009$ for total testosterone and $p=0.010$ for free testosterone) post- $1 \mathrm{~h}$ comparing to baseline concentrations only for RPE6 session. BDNF concentration was higher $(p=0.002)$ post- $0 \mathrm{~h}$ only for the all-out session when comparing to baseline concentrations. However, BDNF was significantly lower post-24 $\mathrm{h}$ after the all-out $(p=0.042)$ and RPE6 $(p=0.032)$ sessions, when compared to baseline values.

Figure 3 shows the percentage of change in creatine kinase concentration for each FFT session and the control session. The percentage of change post- $1 \mathrm{~h}$ was significantly higher for all-out session compared to RPE6 session $(p=0.047)$. No other differences were observed in creatine kinase percentage change between all-out and RPE6 sessions. Effect size (ES) of the changes post-0 $h$ are 0.00 for control, 0.65 for all-out and 0.28 for RPE6; ES Post- $1 \mathrm{~h}$ are 0.02 for control, 0.69 for all-out and 0.14 for RPE6; ES Post-2 h are 0.01 for control, 0.54 for all-out and 0.24 for RPE6; ES Post- $24 \mathrm{~h}$ are 0.13 for control, 0.85 for all-out and 0.51 for RPE6.

For cortisol concentrations, RPE6 and all-out sessions presented a significantly higher percentage change post-0 h compared to control session (Fig. 4). Effect size (ES) of the changes post- $0 \mathrm{~h}$ are 0.13 for control, 1.07 for all-out and 1.34 for RPE6; ES Post-1 h are 0.68 for control, 
Table 1 Creatine kinase and hormonal responses pre- and post-functional fitness sessions with self-regulation of intensity (RPE6) and ALL-OUT and control session [mean (95\% CI)]

\begin{tabular}{|c|c|c|c|c|c|}
\hline & Pre & Post-0 h & Post-1 h & Post-2 h & Post-24 h \\
\hline \multicolumn{6}{|l|}{ Control } \\
\hline Creatine kinase, $\mathrm{U} / \mathrm{L}$ & $282(159-404)$ & $281(161-402)$ & $287(149-424)$ & $279(151-407)$ & $270(134-405)$ \\
\hline Cortisol, ug/dL & $8.9(7.0-10.7)$ & $8.7(7.0-10.5)$ & $7.6(6.4-8.9)$ & $8.3(7.2-9.4)$ & $7.1(6.1-8.1)$ \\
\hline Total testosterone, $\mathrm{ng} / \mathrm{dL}$ & $571(496-646)$ & $583(503-663)$ & $534(456-611)$ & $565(497-633)$ & $526(455-597)$ \\
\hline Free testosterone, ng/dL & $20.3(17.6-23.0)$ & $20.9(18.3-23.5)$ & $18.7(16.1-21.4)$ & $19.6(17.5-21.7)$ & $18.3(16.2-20.3)$ \\
\hline $\mathrm{BDNF}, \mathrm{pg} / \mathrm{mL}$ & $374(167-581)$ & $291(67-650)$ & $344(31-669)$ & $508(122-898)$ & $239(167-311)$ \\
\hline \multicolumn{6}{|l|}{ RPE6 } \\
\hline Creatine kinase, U/L & $318(100-536)$ & $410(111-710)^{\dagger}$ & $359(126-592)^{\dagger}$ & $392(143-641)^{\dagger}$ & $490(192-788)$ \\
\hline Cortisol, ug/dL & $11.0(7.6-14.4)$ & $16.7(12.4-21.0)^{\dagger}$ & $14.6(9.7-19.6)$ & $10.3(5.6-15.0)$ & $8.8(7.0-10.7)$ \\
\hline Total testosterone, $\mathrm{ng} / \mathrm{dL}$ & $514(385-644)$ & $604(460-748)^{\dagger}$ & $460(332-587)^{\dagger}$ & $535(408-663)$ & $544(474-614)$ \\
\hline Free testosterone, ng/dL & $18.3(13.6-22.9)$ & $21.5(16.3-26.7)^{\dagger}$ & $16.3(11.8-20.9)^{\dagger}$ & $19.0(14.4-23.7)$ & $19.5(17.0-22.0)$ \\
\hline $\mathrm{BDNF}, \mathrm{pg} / \mathrm{mL}$ & $254(64-445)$ & $565(151-978)$ & $437(134-740)$ & $332(51-613)$ & $97(45-151)$ \\
\hline \multicolumn{6}{|l|}{ ALL-OUT } \\
\hline Creatine kinase, U/L & $259(169-350)$ & $346(219-474)^{\dagger}$ & $356(236-476)^{\dagger}$ & $332(196-467)^{\dagger}$ & $456(181-731)$ \\
\hline Cortisol, ug/dL & $13.9(9.5-18.4)^{*}$ & $19.7(14.9-24.5)^{\dagger}$ & $19.5(13.9-25.1)^{\dagger}$ & $15.1(11.4-18.8)$ & $8.2(6.7-9.7)^{\dagger}$ \\
\hline Total testosterone, $\mathrm{ng} / \mathrm{dL}$ & $526(491-562)$ & $610(539-682)^{\dagger}$ & $493(465-522)$ & $504(480-528)$ & $570(525-614)$ \\
\hline Free testosterone, ng/dL & $18.3(16.7-20.0)$ & $20.7(18.8-22.7)^{\dagger}$ & $17.3(15.7-18.8)$ & $17.1(16.0-18.2)$ & $19.9(18.7-20.9)$ \\
\hline $\mathrm{BDNF}, \mathrm{pg} / \mathrm{mL}$ & $298(36-559)$ & $632(410-855)^{\dagger}$ & $478(173-783)$ & $180(41-319)$ & $97(55-139)$ \\
\hline
\end{tabular}

BDNF, Brain-Derived Neurotrophic Factor; *Significantly different from control session $(p \leq 0.05)$; ${ }^{\dagger}$ Significantly different from pre $(p \leq 0.05)$

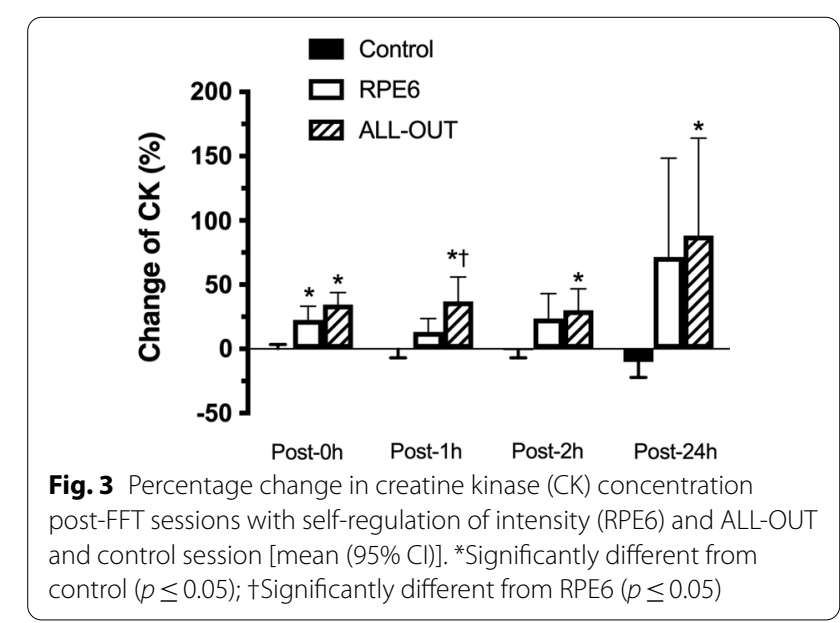

0.81 for all-out and 0.73 for RPE6; ES Post- $2 \mathrm{~h}$ are 0.35 for control, 0.21 for all-out and 0.18 for RPE6; ES Post-24 h are 0.65 for control, 1.31 for all-out and 0.67 for RPE6. The percentage change for total testosterone and free testosterone was significantly different $(p \leq 0.005$ for total testosterone and $p=0.003$ for free testosterone) only post- $2 \mathrm{~h}$ between RPE-6 and all-out (Fig. 5). The Effect size (ES) of the changes for testosterone post- $0 \mathrm{~h}$ was 0.17 for control, 1.26 for all-out and 0.57 for RPE6; ES Post- $1 \mathrm{~h}$ are 0.40 for control, 1.02 for all-out and 0.37 for RPE6; ES Post- $2 \mathrm{~h}$ are 0.05 for control, 0.71 for all-out and 0.14

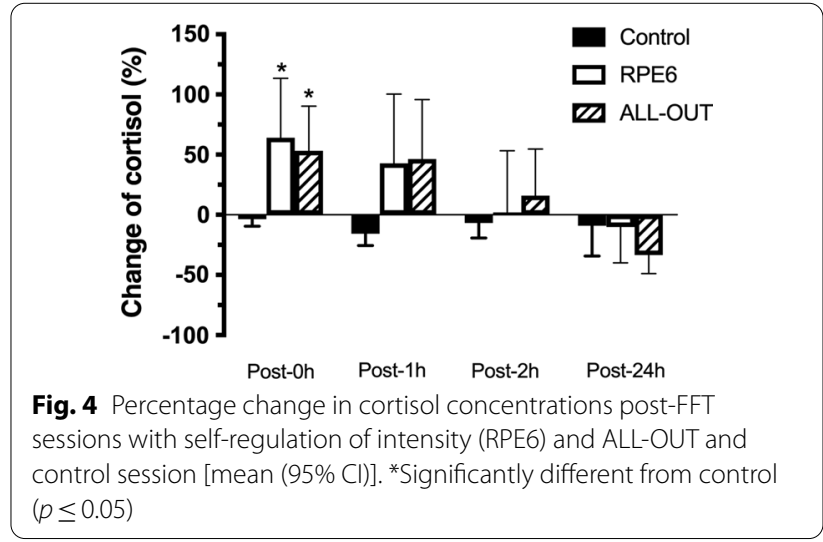

for RPE6; ES Post-24 h are 0.40 for control, 0.88 for allout and 0.21 for RPE6. No statistically significant differences $(p>0.05)$ were observed in the percentage change after the sessions for BDNF (Fig. 6). Effect size (ES) of the changes post- $0 \mathrm{~h}$ are 0.20 for control, 1.33 for all-out and 0.59 for RPE6; ES Post-1 h are 0.07 for control, 0.67 for all-out and 0.56 for RPE6; ES Post- $2 \mathrm{~h}$ are 0.42 for control, 0.40 for all-out and 0.05 for RPE6; ES Post-24 h are 0.27 for control, 1.21 for all-out and 1.33 for RPE6.

There were no significant correlations observed between blood lactate concentration or RPE at the end of the functional fitness sessions and creatine kinase, free testosterone, or total testosterone in the $24 \mathrm{~h}$ 


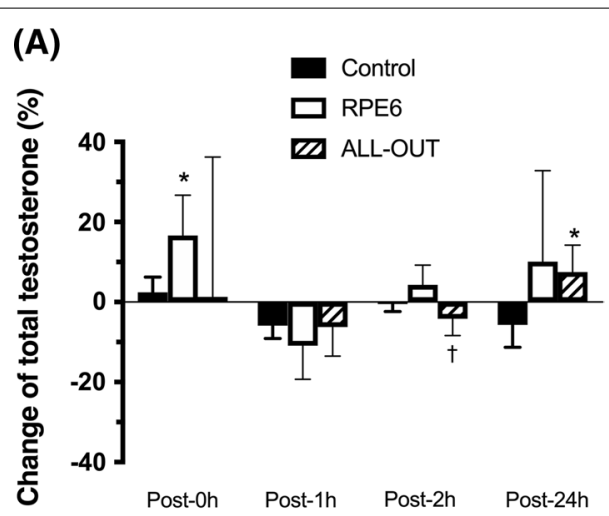

(B)

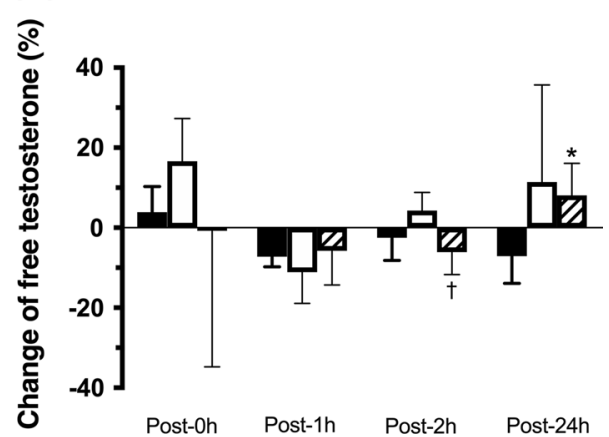

Fig. 5 Percentage of change of total testosterone $(\mathbf{A})$ and free testosterone (B) post-functional fitness sessions with self-regulation of intensity (RPE6) and ALL-OUT and control session [mean (95\% Cl)]. *Significantly different from control ( $p \leq 0.05)$; †Significantly different from RPE6 $(p \leq 0.05)$

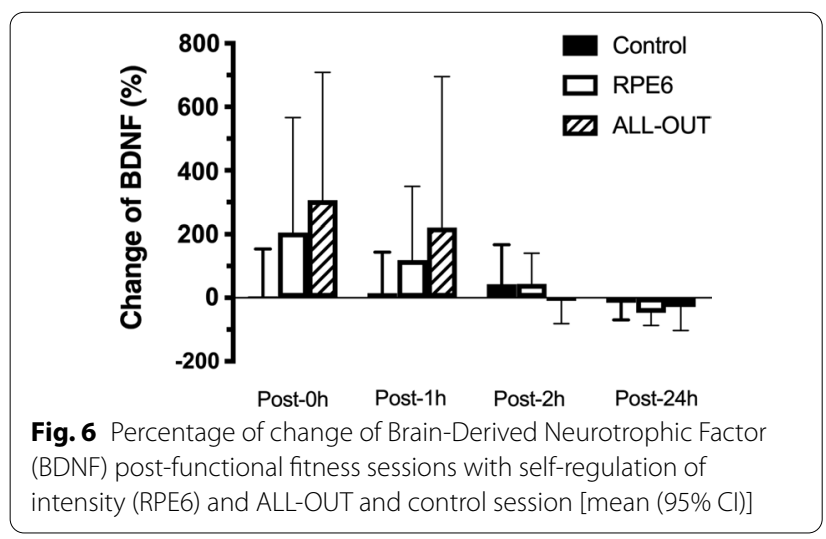

after functional fitness sessions. However, cortisol and BDNF concentrations after the FFT sessions were significantly correlated with blood lactate concentration, and RPE (Table 2).

\section{Discussion}

The major new finding from this study was that BDNF concentrations were greater post-exercise for the all-out session only. Similarly, the percentage change in creatine kinase at different time points after the sessions was only consistently higher than the control condition in the allout session. These results suggest that intensity is a key factor in determining the responses elicited by FFT sessions. As the metabolic conditioning sessions of FFT are usually performed as "all-out" efforts, these results demonstrate that these types of sessions elicit a high level of hormonal and metabolic stress, and that manipulating the intensity of the metabolic conditioning sessions through the use of perceived exertion can lead to lower levels of hormonal and metabolic stress, potentially preventing negative outcomes associated with too much intense training, such as non-functional overreaching or overtraining.

Exercise-induced muscle damage (EIMD) can be attributed to the performance of unaccustomed exercise, or when the intensity, volume, and duration of the training stimulus is excessive to the participant, especially in poorly trained individuals [25]. Amongst other things, EIMD increases cell membrane permeability to muscle enzymes [26], causing leakage which is reflected in increased levels of several metabolic molecules in the interstitial fluid and blood [27, 28]. Furthermore, it is well known that all-out effort muscular work can potentially lead to muscle fiber impairment, that is correlated to increased serum creatine kinase concentration, with previous FFT studies demonstrating elevated creatine kinase levels post all-out sessions. Timón et al. [29] analyzed creatine kinase concentrations in two different training sessions, with the workout 1 consisting of as many rounds as possible of burpees and toes to bar, with the number of repetitions increasing for five minutes. Workout 2 consisted of three rounds of 20 repetitions of wall ball $(9 \mathrm{~kg})$ and 20 repetitions of power cleans in the shortest possible time. The creatine kinase post$24 \mathrm{~h}$ after training was approximately 673 and $864 \mathrm{U} / \mathrm{L}$ for workouts 1 and 2, respectively. Gomes et al. [30] also evaluated creatine kinase concentrations following a single workout ('Cindy' - as many rounds as possible of 5 pull-ups, 10 push-ups, and 15 air squats in 20-min), and showed that creatine kinase concentrations increased post exercise ( 174.9 to $226.7 \mathrm{U} / \mathrm{L}$ ) and remained elevated post-24 h $(\sim 270 \mathrm{U} / \mathrm{L})$. The increase in the concentration occurred even though no external load was utilized, highlighting that the overall intensity of the session might be the key factor for creatine kinase changes.

In fact, when the intensity was likely reduced, the creatine kinase response to exercise was affected. Tibana et al. [11] analyzed the time-course response of creatine 
Table 2 Correlations between cortisol and BDNF concentrations after the functional fitness sessions and blood lactate concentration, and ratings of perceived exertion after the functional fitness sessions

\begin{tabular}{lllll}
\hline & Post-0 $\mathbf{h}$ & Post-1 h & Post-2 h & Post-24 h \\
\hline Cortisol & & & & \\
$\quad$ Blood lactate concentration & $r=0.58 ; p \leq 0.01^{*}$ & $r=0.53 ; p \leq 0.01^{*}$ & $r=0.35 ; p=0.09$ & $r=0.15 ; p=0.48$ \\
$\quad \begin{array}{l}\text { Ratings of perceived exertion } \\
\text { BDNF }\end{array}$ & $r=0.75 ; p \leq 0.01^{*}$ & $r=0.71 ; p \leq 0.01^{*}$ & $r=0.54 ; p \leq 0.01^{*}$ & $r=0.21 ; p=0.33$ \\
$\quad$ & & & \\
Blood lactate concentration & $r=0.51 ; p=0.01^{*}$ & $r=0.47 ; p=0.02^{*}$ & $r=-0.18 ; p=0.40$ & $r=-0.57 ; p \leq 0.01^{*}$ \\
Ratings of perceived exertion & $r=0.47 ; p=0.02^{*}$ & $r=0.34 ; p=0.10$ & $r=-0.32 ; p=0.13$ & $r=-0.55 ; p \leq 0.01^{*}$ \\
\hline
\end{tabular}

BDNF, Brain-Derived Neurotrophic Factor; * statistically significant correlation

kinase following a FFT competition, where athletes were part of a team of three competitors. The results showed no statistical difference in creatine kinase concentration from baseline to post-competition. The fact that during team competitions in FFT the athletes perform a lower volume of repetitions when compared to individual competitions is a potential explanation for these results. In agreement with Tibana et al. [11], our results found a significant increase on creatine kinase percentage change post-24 h only after the all-out protocol. It is possible then, that a FFT session performed at a lower intensity (RPE6) can provide an adequate training stimulus, with a lower stress level to the participants. This would allow practitioners to manage the athletes' training load throughout a training period, ensuring proper recovery during the week of training, potentially minimizing the negative effects associated with frequent all-out bouts of metabolic conditioning [6].

In general, the hormonal acute response is dependent upon the exercise intensity and is the most critical element to tissue remodeling [31]. In the male population, testosterone is a potent anabolic hormone that mediates protein accretion and enhances neural function [32]. A striking finding of the current study was that the total and free testosterone concentrations were lower post- $2 \mathrm{~h}$ for all-out when compared to RPE6 session, suggesting a reduction in the secretory capacity linked to the gonadotropin action. Considering that the all-out session is characterized by greater energy demand and a higher level of neuromuscular fatigue, with the musculature often taken to the point of muscle failure, it is possible that this type of session needs a longer recovery before homeostasis can be restored and hormonal levels can be adjusted. Overall, the all-out session appeared to disrupt hormonal balance immediately after the session, and $2 \mathrm{~h}$ was not sufficient to restore testosterone levels. Nevertheless, the increased testosterone levels post- $24 \mathrm{~h}$ compared to immediately after the sessions (post- $0 \mathrm{~h}$ ), may reflect a compensatory mechanism in response to the testosterone alteration in previous time-points.
Cortisol levels increased immediately post-session in both the RPE6 and all-out conditions, with no difference between sessions. This increase is likely related to enhanced glycogenolysis, gluconeogenesis, and protein catabolism to mobilize fuels for recovery and regeneration after exercise [33]. When investigating the timecourse response of physiological, psychological and performance markers following a FFT competition, Tibana et al. [11] showed a significant decrease in cortisol concentrations after $48 \mathrm{~h}$ when compared to their pre-competition levels, indicating that cortisol release might have a later onset. Additionally, the authors did not observe any correlations between hormonal concentrations, metabolic responses, and immune variables with performance changes (countermovement jump), reinforcing the idea that cortisol changes in the short term are limited.

Prior studies showed that different exercise protocols can act as a stimulus to the hypothalamic-pituitaryadrenocortical axis, which in turn lead to increases in circulating cortisol levels [4, 34]. However, evidence suggests that cortisol levels increase at a rate relatively proportional to the exercise intensity yet reach a final level dependent upon the athlete's training status, total duration of the exercise session, and the hormonal half-life [35-37]. Previous findings support this assertion. Jacks et al. [34] demonstrated that exercise sessions that lasted less than $40 \mathrm{~min}$ in duration elicited no significant differences in cortisol concentrations regardless of their intensity. Likewise, well trained individuals can tolerate a higher intensity of exercise prior to seeing an increase in cortisol concentrations, [36], which might partially explain our findings. Moreover, the release of cortisol typically is upstream of the immune system response [37]. Cortisol is also known to have potent anti-inflammatory effects, considering its role in maintaining neutrophilia, lymphopenia and cytokines bioavailability, which suggests that this hormone has a variety of effects on different functions [37].

Previous investigations that explored the BDNF kinetics after diverse exercise protocols have demonstrated 
acute transient increases in circulating levels during highintensity when compared with low-intensity exercise [38, 39], with the highest concentrations occurring immediately post exercise. To the best of our knowledge, the present study is the first to analyze the role of the intensity on time-course changes in peripheral BDNF levels following FFT sessions. The current findings indicate that BDNF was greater $(p=0.002$; $E S=1.33)$ post- 0 h only for all-out session when compared to baseline concentrations, suggesting that intensity can modulate the BDNF amplitude response caused by FFT. Corroborating this finding, we found a positive correlation between blood lactate concentration during the sessions and BDNF values (post- $0 \mathrm{~h}: \mathrm{r}=0.51 ; \mathrm{p}=0.01^{*}$; post- $\mathrm{h}: \mathrm{r}=0.47$; $p=0.02$ "). Previous studies proposed that tropomyosin receptor kinase $\mathrm{B}$ receptor [40], $\mathrm{Ca}^{+}{ }^{+}$-stimulated intracellular signaling [41] and lactate concentrations [42] can modulate BDNF mRNA levels, which favors positive effects on neuroplasticity.

In a recent review, Müller et al. [43] highlighted a potential regulatory mechanism for the relationship between lactate and BDNF levels in response to exercise. It is well established that lactate promotes plasticity by potentiating NMDA glutamate receptor activity in neurons. Moreover, lactate upregulated intracellular NADH and calcium levels, which consequently can induce BDNF activation. This potential molecular basis to explain the contribution of the BDNF signaling pathway induced by lactate from astrocytes [43]. Moreover, lactate modulates PGC1 $\alpha /$ FNDC5/BDNF pathway in response to exercise through SIRT1 activation [44]. However, while cortisol released during intense exercise might inhibit BDNF synthesis [44], the present study did not find a relationship between BDNF and cortisol responses. Further investigations are required to explain this result. The increases in BDNF concentration despite the changes in cortisol might algo suggest that other adjacent molecular pathways were involved in the changes in BDNF concentrations.

Recently, Ben-Zeev et al. [45] showed that a 3-month FFT program in middle-school adolescents was able to enhance short-term spatial learning, visual pattern separation, and inhibitory control. When comparing a FFT program to a walking intervention and a control group, Wilke et al. [46] suggested that FFT was more effective in improving working memory when compared to low intensity cardiovascular exercise. Thus, it is possible that the increase in BDNF levels after the all-out session reported in the present study can be one of the elements that links exercise to cognitive benefits [46].

Despite the interesting results of this study, some limitations need to be mentioned. First, the findings are limited to a relatively small $(n=8)$ sample of convenience, our specific athlete characteristics, and time frame. Moreover, the Tibana test does not contain all gymnastics movement, weightlifting and powerlifting exercises that are usually performed during the metabolic conditioning of FFT session. Future studies should include the investigation of regulatory molecules (e.g. catecholamine, neurotransmitters and glucocorticoid receptors) and immune variables that participate directly in the increased hormonal and metabolic responses following exercise in order to clarify adjacent mechanisms. In addition, whether longer periods of FFT sessions performed at different intensities (RPE6 and ALL-OUT, for example) will continue to produce further hormonal and metabolic adaptations, and if these changes are associated with modifications in muscle properties, strength, and functional ability remains a provocative hypothesis for further investigation.

\section{Conclusion}

Taken together, the results demonstrate that the intensity at which the metabolic conditioning of FFT sessions are performed has a significant effect on hormonal and metabolic concentrations following exercise. All-out efforts can increase the acute concentration of BDNF and creatine kinase, while leading to an acute reduction in testosterone levels that return to baseline levels, and above, $24 \mathrm{~h}$ post training. When sessions are performed at a lower intensity (RPE6), a rapid recovery of physiological stress markers is seen when compared to an all-out session. The results also allow coaches and practitioners to improve their training programs based on the changes in hormonal and metabolic responses due to alterations in the intensity of the sessions. Future studies should evaluate the chronic effects of different training intensities in the markers of overreaching and overtraining syndrome in previously trained subjects.

\section{Abbreviations \\ AMRAP: As many rounds as possible; CK: Creatine kinase; BDNF: Brain derived neurotrophic factor; EIMD: Exercise-induced muscle damage; FFT: Functional- Fitness; RPE6: Self-regulation training intensity.}

\section{Acknowledgements \\ We are grateful to all subjects who gave of their time and effort to participate in this study.}

\section{Authors' contributions}

RAT, JP and FAV conceived and designed research. RAT, WMS and JHFN conducted experiments. RAT, IVSN, NMFS analyzed data. RAT, IVSN, FHD and MDK wrote the manuscript. All authors read and approved the manuscript.

\section{Funding}

This research received no external funding.

\section{Availability of data and materials}

The datasets used and/or analyzed during this study are not publicly available because the authors do not have permission from the participants to publicly 
share their individual data, but are available from the corresponding author on reasonable request declarations.

\section{Declarations}

\section{Ethics approval and consent to participate}

This study was performed in accordance with the Declaration of Helsinki and approved by University Research Ethics Committee for Human Use (2.698.225/ Universidade Estácio de Sá/UNESA/RJ and ethics ID Pro00110581) and conformed to the Helsinki Declaration on the use of human participants for research. All participants were informed of the experimental procedures and risks and provided their written informed consent prior to the participation in the study.

\section{Consent for publication}

Not applicable.

\section{Competing interests}

The authors declare that they have no competing interests.

\section{Author details}

${ }^{1}$ Graduate Program in Health Sciences, Faculty of Medicine, Federal University of Mato Grosso (UFMT), Cuiabá, Brazil. ' ${ }^{2}$ Laboratory of Molecular Analysis, Graduate Program of Sciences and Technology of Health, University of Brasilia, Brasilia, Brazil. ${ }^{3}$ Laboratory of Exercise Physiology, Faculty Estacio of Vitoria, Vitoria, Brazil. ${ }^{4}$ Graduate Program On Physical Education, Catholic University of Brasilia, Brasilia, Brazil. ${ }^{5}$ Athlete Health Lab, University of Alberta, Edmonton, $A B$, Canada. ${ }^{6}$ Laboratory of Sport and Exercise Psychology, Human Movement Sciences Graduate Program, College of Health and Sport Science of the Santa Catarina State University (UDESC), Florianópolis, Brazil.

Received: 15 June 2021 Accepted: 16 September 2021

Published online: 08 February 2022

\section{References}

1. Tibana RA, Sousa NMF. Are extreme conditioning programmes effective and safe? A narrative review of high-intensity functional training methods research paradigms and findings. BMJ Open Sport Exerc Med. 2018;2(4):e000435.

2. Falk Neto JH, Tibana RA, de Sousa NMF, Prestes J, Voltarelli FA, Kennedy $M D$. Session rating of perceived exertion is a superior method to monitor internal training loads of functional fitness training sessions performed at different intensities when compared to training impulse. Front Physiol. 2020;11:919.

3. Tibana RA, de Almeida LM, Frade de Sousa NM, Nascimento DDC, Neto IVS, de Almeida JA, de Souza VC, Lopes M, Nobrega OT, Vieira DCL, et al. Two consecutive days of crossfit training affects pro and anti-inflammatory cytokines and osteoprotegerin without impairments in muscle power. Front Physiol. 2018;9:771.

4. Tibana RA, de Sousa NMF, Prestes J, Nascimento DDC, Ernesto C, Falk Neto JH, Kennedy MD, Voltarelli FA. Is perceived exertion a useful indicator of the metabolic and cardiovascular responses to a metabolic conditioning session of functional fitness? Sports (Basel). 2019;7(7):161.

5. Jacob N, Novaes JS, Behm DG, Vieira JG, Dias MR, Vianna JM. Characterization of hormonal, metabolic, and inflammatory responses in CrossFit(R) training: a systematic review. Front Physiol. 2020;11:1001.

6. Crawford DA, Drake NB, Carper MJ, DeBlauw J, Heinrich KM. Validity, reliability, and application of the session-rpe method for quantifying training loads during high intensity functional training. Sports (Basel). 2018;6:84

7. Tibana RA, De Sousa NMF, Prestes J, Voltarelli FA. Lactate, heart rate and rating of perceived exertion responses to shorter and longer duration CrossFit $^{\circledR}$ training sessions. J Funct Morphol Kinesiol. 2018;3:60.

8. Cadegiani FA, Kater CE, Gazola M. Clinical and biochemical characteristics of high-intensity functional training (HIFT) and overtraining syndrome: findings from the EROS study (The EROS-HIFT). J Sports Sci. 2019:37:1296-307.

9. Mate-Munoz JL, Lougedo JH, Barba M, Canuelo-Marquez AM, GuodemarPerez J, Garcia-Fernandez P, Lozano-Estevan MDC, Alonso-Melero R,
Sanchez-Calabuig MA, Ruiz-Lopez M, et al. Cardiometabolic and muscular fatigue responses to different CrossFit(R) workouts. J Sports Sci Med. 2018:17:668-79.

10. Mangine GT, Kliszczewicz BM, Boone JB, Williamson-Reisdorph CM, Bechke EE. Pre-anticipatory anxiety and autonomic nervous system response to two unique fitness competition workouts. Sports (Basel). 2019;7:199.

11. Tibana RA, Prestes J, Des NMF, Des VC, Detn O, Baffi M, Ferreira CES, Cunha GV, Navalta JW, Trombeta J, et al. Time-course of changes in physiological, psychological, and performance markers following a functional-fitness competition. Int J Exerc Sci. 2019;12:904-18.

12. Cadegiani FA, Kater CE. Hormonal aspects of overtraining syndrome: a systematic review. BMC Sports Sci Med Rehabil. 2017;9:14.

13. Mastorakos G, Pavlatou M, Diamanti-Kandarakis E, Chrousos GP. Exercise and the stress system. Hormones (Athens). 2005;4:73-89.

14. Izquierdo M, Ibanez J, Calbet JA, Navarro-Amezqueta I, Gonzalez-Izal M, Idoate F, Hakkinen K, Kraemer WJ, Palacios-Sarrasqueta M, Almar M, Gorostiaga EM. Cytokine and hormone responses to resistance training. Eur J Appl Physiol. 2009;107:397-409.

15. Walsh JJ, Tschakovsky ME. Exercise and circulating BDNF: Mechanisms of release and implications for the design of exercise interventions. Appl Physiol Nutr Metab. 2018;43:1095-104.

16. Knaepen K, Goekint M, Heyman EM, Meeusen R. Neuroplasticity exercise-induced response of peripheral brain-derived neurotrophic factor: a systematic review of experimental studies in human subjects. Sports Med. 2010;40:765-801

17. Murawska-Cialowicz E, Wojna J, Zuwala-Jagiello J. Crossfit training changes brain-derived neurotrophic factor and irisin levels at rest, after wingate and progressive tests, and improves aerobic capacity and body composition of young physically active men and women. J Physiol Pharmacol. 2015:66:811-21.

18. Pereira ES, Krause Neto W, Calefi AS, Georgetti M, Guerreiro L, Zocoler CAS, Gama EF. Significant acute response of brain-derived neurotrophic factor following a session of extreme conditioning program is correlated with volume of specific exercise training in trained men. Front Physiol. 2018;9:823.

19. Reycraft JT, Islam H, Townsend LK, Hayward GC, Hazell TJ, Macpherson REK. Exercise intensity and recovery on circulating brain-derived neurotrophic factor. Med Sci Sports Exerc. 2020;52:1210-7.

20. de Souza RAS, da Silva AG, de Souza MF, Souza LKF, Roschel H, da Silva SF, Saunders B. A systematic review of CrossFit(R) workouts and dietary and supplementation interventions to guide nutritional strategies and future research in CrossFit(R). Int J Sport Nutr Exerc Metab. 2021;31:187-205.

21. Foster C, Florhaug JA, Franklin J, Gottschall L, Hrovatin LA, Parker S, Doleshal P, Dodge C. A new approach to monitoring exercise training. J Strength Cond Res. 2001;15:109-15.

22. Morishita S, Tsubaki A, Takabayashi T, Fu JB. Relationship between the rating of perceived exertion scale and the load intensity of resistance training. Strength Cond J. 2018:40:94-109.

23. Cohen J. Statistical Power Analysis for the Behavioral Sciences: L. Erlbaum Associates; 1988.

24. Hopkins WG, Marshall SW, Batterham AM, Hanin J. Progressive statistics for studies in sports medicine and exercise science. Med Sci Sports Exerc. 2009;41:3-13.

25. Schoenfeld BJ. Does exercise-induced muscle damage play a role in skeletal muscle hypertrophy? J Strength Cond Res. 2012;26:1441-53.

26. Spada TC, Silva J, Francisco LS, Marcal LJ, Antonangelo L, Zanetta DMT, Yu L, Burdmann EA. High intensity resistance training causes muscle damage and increases biomarkers of acute kidney injury in healthy individuals. PLOS ONE. 2018;13:e0205791.

27. Baird MF, Graham SM, Baker JS, Bickerstaff GF. Creatine-kinase- and exercise-related muscle damage implications for muscle performance and recovery. J Nutr Metab. 2012;2012:960363.

28. Kindermann W. Creatine kinase levels after exercise. Dtsch Arztebl Int 2016;113:344.

29. Timon R, Olcina G, Camacho-Cardenosa M, Camacho-Cardenosa A Martinez-Guardado I, Marcos-Serrano M. 48-hour recovery of biochemical parameters and physical performance after two modalities of CrossFit workouts. Biol Sport. 2019;36:283-9.

30. Gomes JH, Mendes RR, Franca CS, Da Silva-Grigoletto ME. Pereira da Silva DR, Antoniolli AR, de Oliveira ESAM, Quintans-Junior LJ: Acute leucocyte, 
muscle damage, and stress marker responses to high-intensity functional training. PLoS ONE. 2020;15:e0243276.

31. Kraemer WJ, Ratamess NA. Hormonal responses and adaptations to resistance exercise and training. Sports Med. 2005;35:339-61.

32. Kraemer WJ, Ratamess NA, Hymer WC, Nindl BC, Fragala MS. Growth hormone(s), testosterone, insulin-like growth factors, and Cortisol: roles and integration for cellular development and growth with exercise. Front Endocrinol (Lausanne). 2020;11:33.

33. O'Leary CB, Hackney AC. Acute and chronic effects of resistance exercise on the testosterone and cortisol responses in obese males: a systematic review. Physiol Res. 2014;63:693-704.

34. Jacks DE, Sowash J, Anning J, McGloughlin T, Andres F. Effect of exercise at three exercise intensities on salivary cortisol. J Strength Cond Res. 2002;16:286-9.

35. Duclos M, Corcuff JB, Rashedi M, Fougere V, Manier G. Trained versus untrained men: different immediate post-exercise responses of pituitary adrenal axis. A preliminary study. Eur J Appl Physiol Occup Physiol. 1997;75:343-50

36. Jurimae J, Jurimae T, Purge P. Plasma testosterone and cortisol responses to prolonged sculling in male competitive rowers. J Sports Sci. 2001;19:893-8.

37. Viru A, Viru M. Cortisol-essential adaptation hormone in exercise. Int J Sports Med. 2004;25:461-4.

38. Schmidt-Kassow M, Schadle S, Otterbein S, Thiel C, Doehring A, Lotsch J, Kaiser J. Kinetics of serum brain-derived neurotrophic factor following low-intensity versus high-intensity exercise in men and women. NeuroReport. 2012;23:889-93.

39. Antunes BM, Rossi FE, Teixeira AM, Lira FS. Short-time high-intensity exercise increases peripheral BDNF in a physical fitness-dependent way in healthy men. Eur J Sport Sci. 2020;20:43-50.

40. Kim HJ, Song BK, So B, Lee O, Song W, Kim Y. Increase of circulating BDNF levels and its relation to improvement of physical fitness following 12 weeks of combined exercise in chronic patients with schizophrenia: a pilot study. Psychiatry Res. 2014;220:792-6.

41. Zheng F, Zhou X, Moon C, Wang H. Regulation of brain-derived neurotrophic factor expression in neurons. Int J Physiol Pathophysiol Pharmacol. 2012;4:188-200.

42. Sobral-Monteiro-Junior R, Maillot P, Gatica-Rojas V, Avila WRM, de Paula AMB, Guimaraes ALS, Santos SHS, Pupe CCB, Deslandes AC. Is the "lactormone" a key-factor for exercise-related neuroplasticity? A hypothesis based on an alternative lactate neurobiological pathway. Med Hypotheses. 2019:123:63-6.

43. Muller P, Duderstadt Y, Lessmann V, Muller NG. Lactate and BDNF: key mediators of exercise induced neuroplasticity? J Clin Med. 2020;9:1136.

44. Garcia-Suarez PC, Renteria I, Moncada-Jimenez J, Fry AC, JimenezMaldonado A. Acute systemic response of BDNF, Lactate and cortisol to strenuous exercise modalities in healthy untrained women. Dose Response. 2020;18:1559325820970818.

45. Ben-Zeev T, Hirsh T, Weiss I, Gornstein M, Okun E. The effects of Highintensity Functional Training (HIFT) on spatial learning, visual pattern separation and attention span in adolescents. Front Behav Neurosci. 2020;14:577390

46. Wilke J, Giesche F, Klier K, Vogt L, Herrmann E, Banzer W. Acute effects of resistance exercise on cognitive function in healthy adults: a systematic review with multilevel meta-analysis. Sports Med. 2019;49:905-16.

\section{Publisher's Note}

Springer Nature remains neutral with regard to jurisdictional claims in published maps and institutional affiliations. 